\title{
Users Can Do Better with PDAs Than Paper: A Usability Study of PDA-Based vs. Paper-Based Nursing Documentation Systems
}

\author{
Néstor J. Rodríguez, José A. Borges, Gilberto Crespo, Carlos Pérez, \\ Carlos Martinez, Celia R. Colón-Rivera, and Aixa Ardín \\ Institute for Computing and Informatics Studies \\ University of Puerto Rico - Mayagüez \\ \{nestor, borges\} @ece. uprm. edu, \\ \{gilberto.crespo, bigbadboston\} @gmail.com, \\ martinez_carlosj@yahoo.com, celiacr@ece.uprm.edu, \\ aixaardin@gmail.com
}

\begin{abstract}
Personal Digital Assistants (PDAs) are a viable technology for providing access to Electronic Medical Records at the point-of-care. However, acceptance of this technology by clinicians will depend on how easy they can make the transition from the system they normally use to a PDA-based system. Since many hospitals are still using paper-based patient record systems this study intends to provide some insight on the aspects that need to be considered in the transition from a paper-based system to a PDA-based system. The study compares the interaction of nurses with PDA-based and paper-based nursing documentation systems in terms of performance and subjective satisfaction. Twenty staff nurses from a metropolitan hospital performed twelve tasks on each system. The study supports the conclusion that a PDA-based nursing documentation system can be superior to a paper-based system in term of performance for tasks that don't required writing notes. Nurses were significantly more satisfied with the PDA-based system than with the paper-based system with every interaction and system aspect evaluated on the study. In general the results of the study provide hard evidence to predict an easy transition for nurses from a paper-based system to a PDA-based system nursing documentation system.
\end{abstract}

Keywords: mobile devices, PDAs, usability, medical informatics.

\section{Introduction}

As health care grows more complex, the access to timely information, and the ability to communicate effectively about patient care are more important than ever. However, in spite of the proven and potential benefits of Electronic Medical Records (EMRs) regarding those issues, paper-based records are still predominant. Factors such as cost, user resistance, complexity and poor usability, have contributed to the slow implantation of these systems. This is especially true with regard to information 
systems to support nursing activities, point-of-care support, and nursing documentation. As stated by Ammenwerth [1], the documentation of the nursing process is an important but often neglected part of clinical documentation.

Within a clinical setting nurses are key collectors, generators, and users of patient information, and good nursing care is dependent upon the quality of the information they have available [2,3]. Paper-based records are frequently illegible, difficult to search, fragmented across sites of care, and limited in their ability to provide decision support [10]. They are also cumbersome for supporting care coordination, since they cannot be accessed remotely, and are available to only one person at a time. As stated by Rinkus [6], paper-based medical records often lack means to document key nursing activities.

An Electronic Medical Record can help overcome many of the problems associated with paper-based patient record systems. However, since most of the patients' information is collected at bedside, EMRs can be greatly improved with mobile technology. The portability of Personal Digital Assistants (PDAs) and their ability to link to a central database without a hard-wired connection make them an alternative for providing access to EMRs at the point-of-care. However, there are limitations such as screen size and input modalities that can affect the acceptability and usability of PDAs.

Several studies have documented these problems and in some cases demonstrated poor acceptance [5] and usage barriers [4] of PDAs by clinicians, due in part to its input modalities. A usability study by N. J. Rodriguez [7] found that physicians are faster on laptops than on PDAs when performing tasks such as text entry and reading; although they are faster on PDAs when performing tasks that only require pointing and clicking. Another study by Young 11] described an evaluation made by nurses on a hand-held device for data entry on nursing record simulations. Nurses considered that the pen-based interface was less desirable for entering textual data than the keyboard-based interface. They thought that the data was less accurate and the data entry process was difficult. Yet another usability study of nurses interacting with PDAs and Laptops [8] demonstrated that writing notes is significantly slower on PDAs as compared to Laptops. Even though PDAs are not very efficient for writing notes the Rodriguez's study [8] demonstrated that PDAs are a viable technology for supporting most nursing documentation tasks. That study revealed that nurses can be as effective with PDAs as with Laptops in performing typical nursing documentation tasks other than writing notes.

Most of the studies cited in the previous paragraph compared PDA-based systems with other electronic-based systems. However, no formal study is found in the literature that compares nurses' interaction with paper-based and PDA-based nursing documentation systems. The study described in this article is the first of its kind. It is a usability study that compares nurses' interaction with PDA-based and paper-based nursing documentations systems in terms of performance (completion time and number of tasks completed) and user satisfaction. The objective of the study was to get some insight on the aspects that need to be considered in the transition from a paperbased system to a PDA-based system. 


\section{Methods}

\subsection{The Systems}

One of the systems use for the study was a paper-based nursing documentation system of a cardiovascular hospital. The other system was a PDA nursing documentation application designed for the same hospital and with similar functionality. Both versions facilitate the documentation of typical nursing tasks such as handling physicians' orders, medication administration, drip type medication administration, vital signs documentation, writing nursing notes, performing daily assessment, collecting I/O measurements and assessment of pain.

The PDA version was implemented on a PDA running the Microsoft Pocket PC operating system. A documentation category is accessed by selecting it from a pulldown menu. The corresponding information is typically displayed in a window in the form of a list (see figure 1.a). Each line of the list corresponds to a previous documentation entry. Detailed information of each entry can be displayed in a new window by clicking on the entry (see figure 1.b). The window of each documentation category provides a $\mathrm{New}$ button at the right side bottom that opens a new window that allows nurses to enter a new documentation entry.

The paper-based system used for the study consisted of the record of hypothetical patients. The record had typical hospital documentation forms, lab results forms and

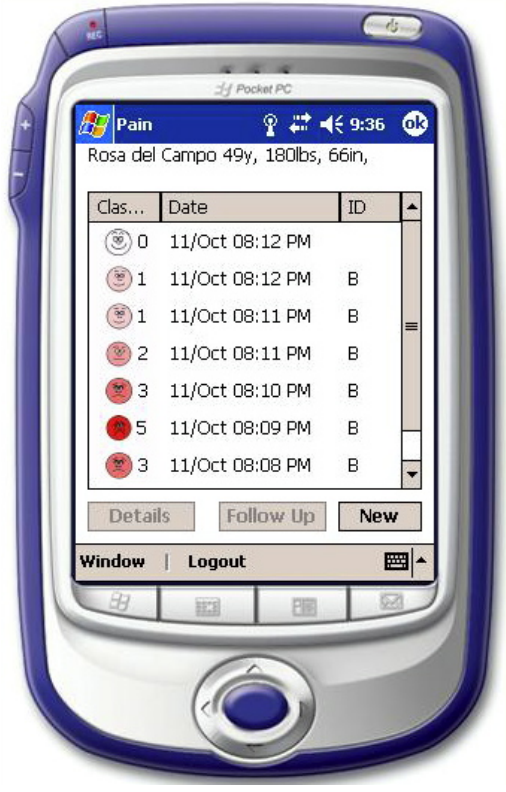

(a) Pain Window

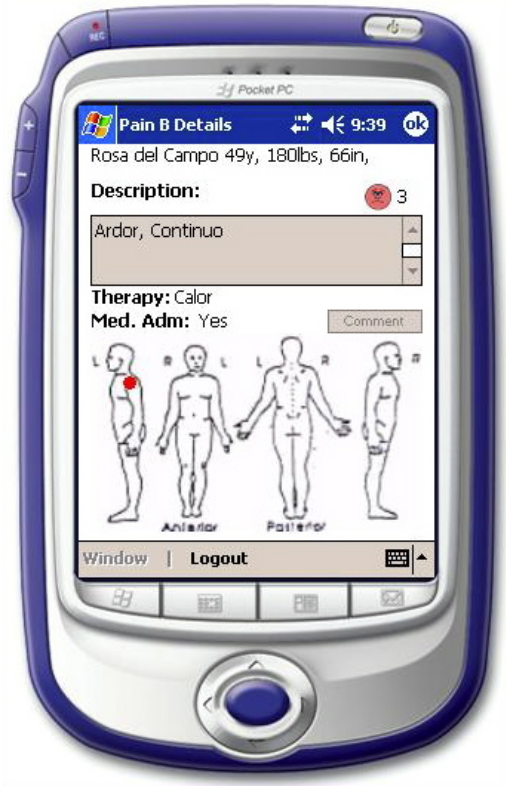

(b) Detail Window

Fig. 1. The PDA nursing documentation user interface 
diagnostic studies forms. The same information was replicated on the PDA version of the record of the same patient.

\subsection{Participants}

Twenty staff nurses from a hospital serving only a cardiovascular clientele participated in the study. They were selected on a first-come first-serve basis from those that responded a call for participation and were compensated for their participation. Their experience as staff nurses ranged from 1 to 27 years $(M=9.3$ years). Experience with computers ranged from 0 to 8 years $(M=4.4$ years). On average, they used computers on their job for 2.7 hours per day. All the participants had experience using the paperbased system. None of them had prior experience with the PDA nursing documentation application, nor with any other electronic patient record system. Also, none of them had experience using PDAs.

\subsection{The Tasks}

Each participant was asked to perform the following twelve tasks for a specific patient:

1. Indicate patient's age and weight.

2. Indicate the most recent registered patient's temperature.

3. Acknowledge any pending medication order as administered.

4. Enter a set of vitals signs

5. Indicate the total balance of intake/output of fluids in the last 24 hours.

6. Look for a specific physician note.

7. Enter a specific patient assessment information.

8. Enter a specific I/O information.

9. Enter a specific text as a note.

10. Enter a specific pain information.

11. Look for most recent physician consult order and acknowledge it as notified by phone.

12. Show where in the record is indicated why a dose of Roboxine was omitted.

\subsection{Research Design}

Before proceeding with the test the participants were asked to sign consent forms and fill out a background questionnaire. They were asked to perform the twelve tasks described in the previous section on the PDA system and the paper-based system. To balance for learning effect, ten of the participants performed the tasks first on the PDA version and then on the paper-based version. The other ten participants performed the tasks first on the paper-based version and then on the PDA version. Participants were given a short tutorial session on the PDA version (about 18 minutes). The tasks on the paper-based system were performed on top of a desk while the tasks on the PDA were performed in a stand up position.

The participants were asked to fill out a subjective user satisfaction questionnaire. The questionnaire asked the participants to rate nine interaction aspects and five system aspects of each system using a 1-7 scale (1 being poor and 7 being excellent). 


\subsection{Statistical Analysis}

The dependent variables of this study were task completion time, number of tasks completed, and subjective user satisfaction. An independent-samples $t$ test was used to compare completion times. The Mann-Whitney test with an $\alpha$-level of .05 was used to compare the number of task completed and user satisfaction. Linear regression was used to evaluate the learning effect between the two versions.

\section{Results}

An independent-samples $t$-test did not reveal a significant difference between the average time it took the participants to complete all the tasks on the PDA (Mean = $633.6, S D=187.9)$ and the paper-based system $($ Mean $=723.7, S D=250.2)$. The average times and standard deviations for the completion time of individual tasks are presented in table 1. Independent-samples t-tests revealed significant difference on the average time it took the participants to complete tasks one, two, three, six, nine, ten, and eleven. The participants were significantly faster on the PDA than on the paperbased system in all cases except on task number 9 (writing a note). The differences in average completion time for individual task can be appreciated in figure 2 .

Table 1. Means and standard deviations for completion times of each task for each system

\begin{tabular}{|r|r|r|r|r|}
\cline { 2 - 5 } \multicolumn{1}{c|}{} & \multicolumn{2}{c|}{ PDA } & \multicolumn{2}{c|}{ Paper-based } \\
\hline Tasks & \multicolumn{1}{c|}{ Mean } & \multicolumn{1}{c|}{ SD } & \multicolumn{1}{c|}{ Mean } & \multicolumn{1}{c|}{ SD } \\
\hline & (seconds) & (seconds) & (seconds) & (seconds) \\
\hline 1 & 5.0 & 5.9 & 27.6 & 25.1 \\
\hline 2 & 8.7 & 8.1 & 18.2 & 15.8 \\
\hline 3 & 17.3 & 15.3 & 40.0 & 35.5 \\
\hline 4 & 32.0 & 11.0 & 44.0 & 28.2 \\
\hline 5 & 27.4 & 24.0 & 43.8 & 30.7 \\
\hline 6 & 27.8 & 17.9 & 71.3 & 46.0 \\
\hline 7 & 193.8 & 83.3 & 223.0 & 126.4 \\
\hline 8 & 36.3 & 17.3 & 28.3 & 16.0 \\
\hline 9 & 190.5 & 64.9 & 83.8 & 35.0 \\
\hline 10 & 39.3 & 22.4 & 59.3 & 23.3 \\
\hline 11 & 24.7 & 26.6 & 52.8 & 40.8 \\
\hline 12 & 31.2 & 30.2 & 31.8 & 30.2 \\
\hline
\end{tabular}

The participants were able to complete the majority of the tasks. A Mann-Whitney tests did not reveal significant difference in the total number of tasks completed by the participants. The participants completed an average of 11.1 tasks on the PDA and 11.6 on the paper-based system. No significant differences were found in task completion for individual tasks on the PDA and the paper-based system.

The average rating given to each of the interaction and system aspects of the PDA and the paper-based systems are summarized in figure 3. A Mann-Whitney test did reveal a significant difference in the average overall satisfaction rating given to the 


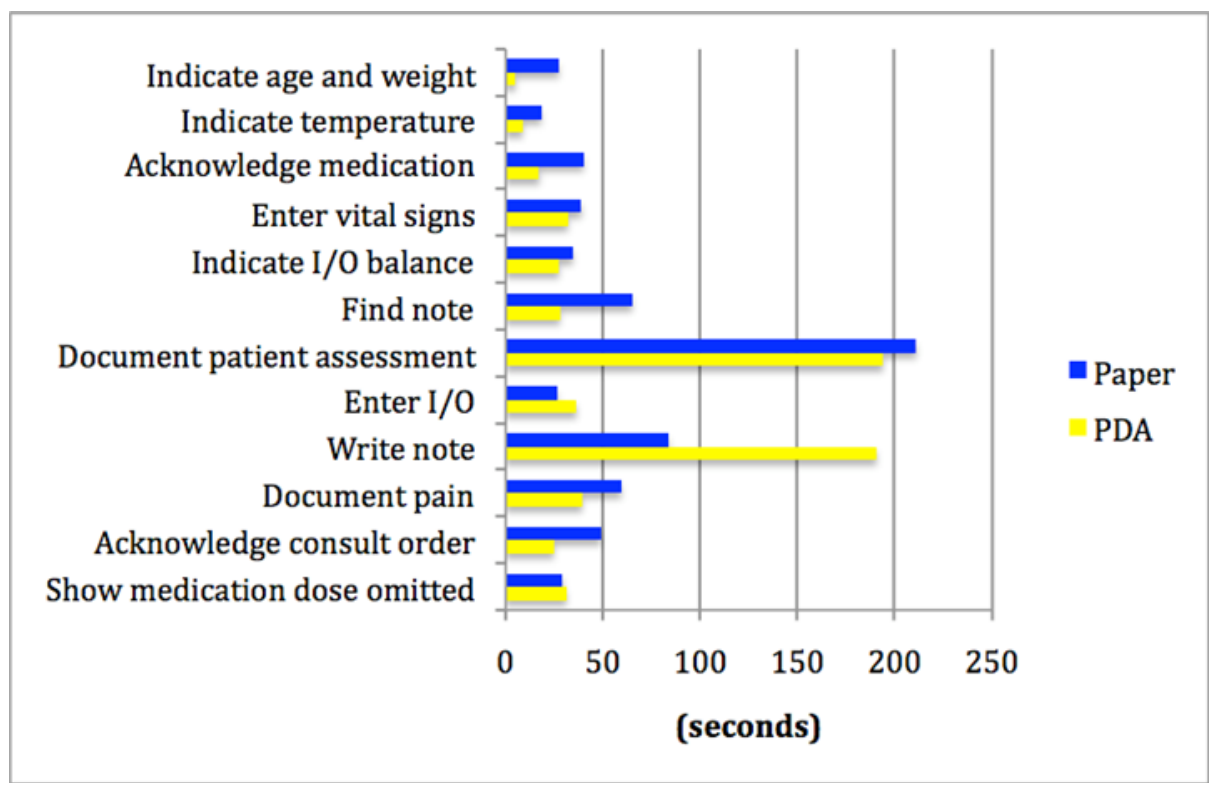

Fig. 2. Average completion time for individual tasks for each system

eight interaction aspects I1-I8. The participants were more satisfied with the PDA system $(M=6.6)$ than with the paper-based system $(M=5.3)$. A Mann-Whitney test also revealed a significant difference on the overall average satisfaction rating given to the 5 system aspects considered (S1-S5). The participants were more satisfied with the PDA system $(M=6.8)$ than with the paper-based system $(M=5.0)$. Significant differences were also found between the PDA and paper-based systems for all individual satisfaction aspects. The participants were more satisfied with the PDA system than with the paper-based system for each of the thirteen individual aspects.

\section{Discussion}

The results of the study indicate that no significant difference exists in the overall time it took nurses to complete tasks with the PDA and with the paper-based system. This result was unexpected because due to the fact that the participants had experience using the paper-based system but did not know the PDA system except for the short tutorial taken prior the test, we expected the nurses to complete the tasks faster with the paper-based system than with the PDA system. These results are similar to the results of a study by N. J. Rodríguez [9] that demonstrated that physicians can be as fast on a paper-based system than on an electronic patient record system. Thus, the results of the present study and of the Rodríguez's study [9] support the assertion that clinicians with little or no experience using an electronic patient record system can perform typical tasks with it as fast as with a paper-based system. 


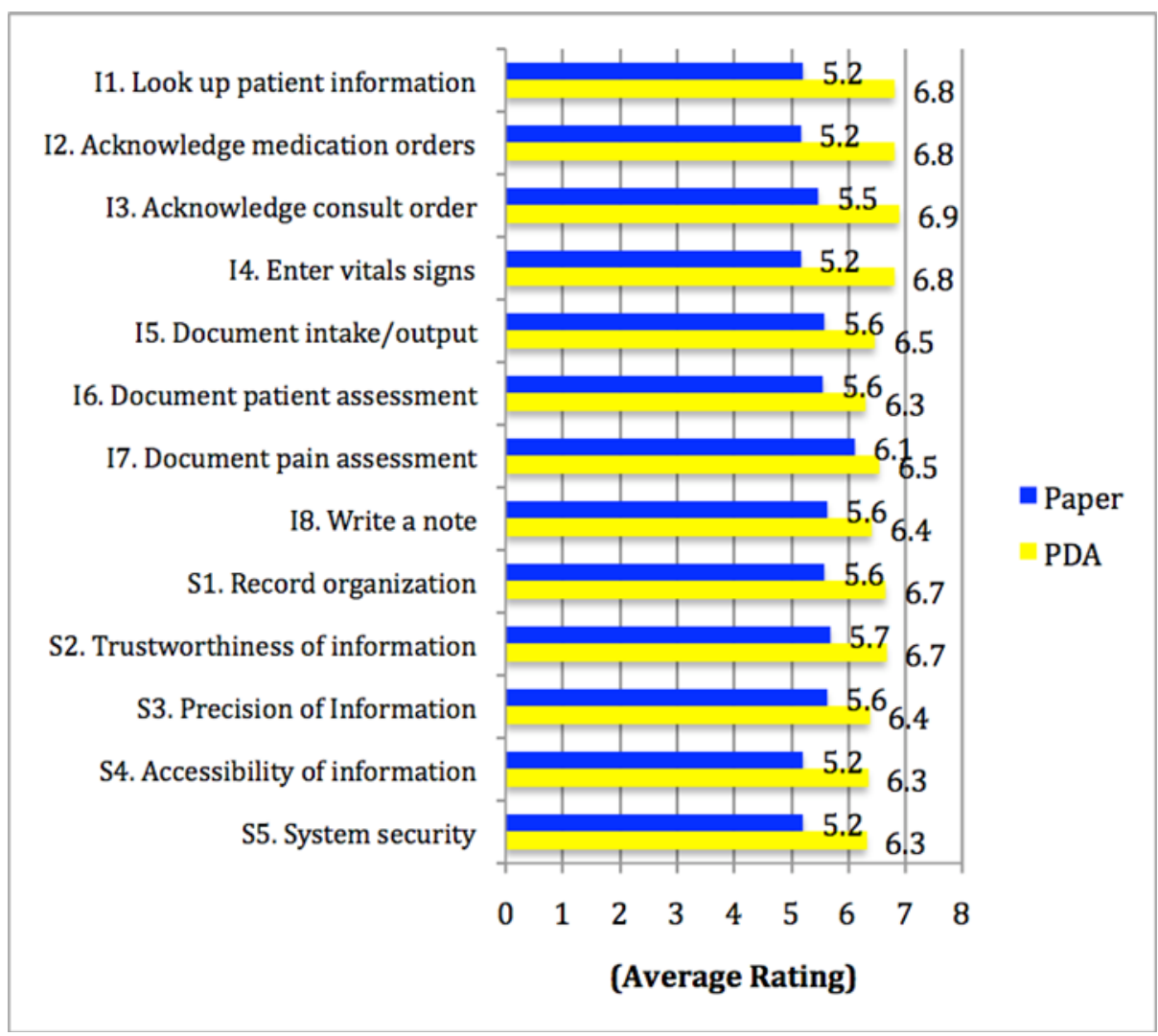

Fig. 3. Average satisfaction ratings for interaction and system aspects of the paper-based and PDA systems

A correlation analysis was performed between the the average overall completion time on each system and the participants' age, years of work experience, years of experience using computers, daily use of computers, daily use of computers on the workplace, and the use of glasses for reading and using computers. The analysis only revealed a correlation between the nurses' age and the overall time it took to complete the tasks on the PDA system. Younger nurses tend to take shorter times in completing tasks on the PDA than older nurses.

The results of time completion for individual tasks shows that the task of writing a note is one of the most time consuming tasks and one with the higher percentage difference. The effect of this task on the overall completion time is very significant. We ran an independent samples t-test of the overall completion time without considering this task and found a significant difference between the PDA system and the paper-based system, $t(38)=3.25, p<.05$. Without considering the task of writing a note the participants completed the tasks on the PDA faster (Mean $=443.2, S D=$ $150.8)$ than on the paper-based system (Mean $=639.9, S D=224.8)$. These results confirm that writing notes on PDAs is significantly more time consuming than writing on paper. This limitation of PDAs for writing notes was also evidenced on a study by 
N. J. Rodriguez et. al. [8] that revealed that writing a note on a PDA was significantly more time consuming than writing it on a laptop.

The results indicate that the participants were significantly faster on the PDA than on the paper-based systems on tasks one, two, three, six, ten, and eleven. This finding is very interesting if we take in consideration that the participants did not have experience with the PDA system, nor using PDAs. For the majority of them the test represented the first time they held a PDA. These results can be attributed to two factors. First, the tasks required searching the paper-based record, which is time consuming in comparison to navigating on the PDA system to perform the tasks. Second, the interfaces of the PDA application exhibited good usability attributes (learnability and efficiency).

Even though the participants did not have experience with the PDA nursing documentation system, nor using PDAs, they were able to complete the large majority of the tasks on both systems. These results indicate that the PDA nursing documentation system is very easy to learn. Thus, nurses should be able to make the transition from a paper-based nursing documentation system to a PDA-based system with minimal training. These finding are very similar to the ones found in the Rodriguez's [8] study. On that study nurses that did not have experience with PDAs were also able to complete most of the nursing documentation tasks after being given very short tutorials.

The results revealed that nurses were significantly mores satisfied with the PDA system than with the paper-based system for overall physical aspects, overall system aspects and all the thirteen individual satisfaction aspects considered for the study. These results are very encouraging because user satisfaction facilitates the transition from an existing system to a new system. The level of satisfaction evidenced by the participants of the study with the PDA nursing documentation system suggest an easy transition from a paper-based system to a PDA-based system exhibiting good usability attributes.

\section{Conclusion}

The results of the study indicate that nurses are as fast on the PDA as on the paperbased nursing documentation system performing typical nursing documentation tasks. However, when the task of writing notes is not considered, nurses can be significantly faster on a PDA system than on a paper-based system. These results support the conclusion that a PDA-based nursing documentation system can be superior to a paperbased system in term of performance for tasks that don't required writing notes.

The study revealed that age is a factor that can influence nurses' performance on the PDA system. In general younger nurses exhibit better performance than older nurses.

The nurses that participated in the study were able to complete most of the tasks with just a short tutorial of the systems and without having any experience using PDAs or electronic patient record systems. Thus, we can conclude that nurses are able to learn to use PDA-based nursing documentation applications with relative ease and minimal training.

Nurses were significantly more satisfied with the PDA system than with the paper-based system for every satisfaction aspects considered for the study. Thus, it is 
possible to design PDA-based nursing documentation systems that generate significantly high levels of satisfaction among nurses.

The performance exhibited by the nurses that participated in the study with the PDA system, the high number of task completed by them and the high level of satisfaction evidenced by them suggests an easy transition for nurses from a paper-based system to a PDA-based system. Nurses can do better with PDA-based than with paper-based nursing documentation systems.

\section{References}

1. Ammenwerth, E., Mansmann, U., Iller, C., Eichstader, R.: Factors Affecting and Affected by User Acceptance of Computer-based Nursing Documentation: Results of a Two-year Study. JAMIA 10, 69-84 (2002)

2. Charters, K., Cappello, C.: Informatics in Clinical Practice. In: Foster, S.D., FaultCallahan, M. (eds.) A Professional Study and Resource Guide for the CRNA, pp. 489-502. American Association of Nurse Anesthetists, Park Ridge (2001)

3. Charters, K.: Nursing Informatics, Outcomes, and Quality Improvement. AACN Clinical Issues: Advanced Practice in Acute and Critical Care 14, 282-294 (2003)

4. Jao, C., Hier, S.: Evaluating a Digital Resident Diagnosis Log: Reasons for Limited Acceptance of a PDA Solution. In: Proc. AMIA Symposium 2003, p. 876 (2003)

5. Lu, Y.C., et al.: Why Don't Physicians Use Their Personal Digital Assistants? In: Proc. AMIA Symposium, p. 405 (2003)

6. Rinkus, S., Chitwood, A.: Cognitive Analyses of a Paper Medical Record and Electronic Medical Record on the Documentation of Two Nursing Tasks: Patient Education and Adherence Assessment of Insulin Administration. In: Proc. AMIA Symp., pp. 657-661 (2002)

7. Rodríguez, N.J., Borges, J.A., Soler, Y., Murillo, V.L., Sands, D.Z.: A usability study of physicians interaction with PDA and laptop applications to access an electronic patient record system. In: 17th IEEE International Symposium on Computer-Based Medical Systems (2004)

8. Rodríguez, N.J., Borges, J.A., Soler, Y., Murillo, V.L., Colón, C.R.: PDA vs. laptop: a comparison of two versions of a nursing documentation application. In: Proc. of the 16th IEEE Symp. on Computer-Based Medical Systems (2003)

9. Rodriguez, N.J., Borges, J.A., Murillo, V.L., Sands, D.Z., Ortiz, J.: A Usability Study of Physicians Interaction with a Paper-Based Patient Record System and a Graphical-Based Electronic Patient Record System. In: Proc. AMIA Symp. 2002 (November 2002)

10. Wilcox, A., Jones, S.S., Dorr, D.A., et al.: Use and impact of a computer-generated patient summary worksheet for primary care. In: AMIA Symp., pp. 824-828 (2005)

11. Young, P.M.C., Leung, R.M.W., Ho, L.M., McGhee, S.M.: An Evaluation of the Use of Hand-Held Computers for Bedside Nursing Care. International Journal of Medical Informatics 62, 189-193 (2001) 